\title{
The band-edge excitons observed in few-layer $\mathrm{NiPS}_{3}$
}

\author{
Ching-Hwa Ho $\mathbb{D}^{1,2 \bowtie}$, Tien-Yao Hsu ${ }^{1,2}$ and Luthviyah Choirotul Muhimmah ${ }^{1}$
}

Band-edge excitons of few-layer nickel phosphorous trisulfide $\left(\mathrm{NiPS}_{3}\right)$ are characterized via micro-thermal-modulated reflectance $(\mu \mathrm{TR})$ measurements from 10 to $300 \mathrm{~K}$. Prominent $\mu$ TR features of the A exciton series and $\mathrm{B}$ are simultaneously detected near the band edge of $\mathrm{NiPS}_{3}$. The $\mathrm{A}$ exciton series contains two sharp $A_{1}$ and $A_{2}$ levels and one threshold-energy-related transition (direct gap, $\left.E_{\infty}\right)$, which are simultaneously detected at the lower energy side of $\mathrm{NiPS}_{3}$. In addition, one broadened $\mathrm{B}$ feature is present at the higher energy side of few-layer $\mathrm{NiPS}_{3}$. The $\mathrm{A}$ series excitons may correlate with majorly $d$-to- $d$ transition in the Rydberg series with threshold energy of $E_{\infty} \cong 1.511 \mathrm{eV}$ at $10 \mathrm{~K}$. The binding energy of $\mathrm{A}_{1}$ is about $36 \mathrm{meV}$, and the transition energy is $A_{1} \cong 1.366 \mathrm{eV}$ at $300 \mathrm{~K}$. The transition energy of $\mathrm{B}$ measured by $\mu \mathrm{TR}$ is about $1.894 \mathrm{eV}$ at $10 \mathrm{~K}$. The excitonic series $\mathrm{A}$ may directly transit from the top of valence band to the conduction band of $\mathrm{NiPS}_{3}$, while the $\mathrm{B}$ feature might originate from the spin-split-off valence band to the conduction band edge. The direct optical gap of $\mathrm{NiPS}_{3}$ is $\sim 1.402 \mathrm{eV}$ at $300 \mathrm{~K}$, which is confirmed by $\mu \mathrm{TR}$ and transmittance experiments.

npj 2D Materials and Applications (2021)5:8; https://doi.org/10.1038/s41699-020-00188-8

\section{INTRODUCTION}

Two-dimensional (2D) semiconductors exhibit immense functionalities and practicalities of large area ${ }^{1,2}$, ultra-thin ${ }^{3,4}$, smooth surface $^{5}$, high carrier mobility ${ }^{6-8}$, flexible layers ${ }^{9-11}$, and thickness-tunable band-gap modulation ${ }^{12,13}$ that have gradually received blooming attention in semiconductor technological studies and development in the post-silicon era. Among the 2D semiconductors, transition-metal dichalcogenides (TMDCs) $\mathrm{MX}_{2}(\mathrm{M}=$ $\mathrm{W}, \mathrm{Mo}, \mathrm{Re}$ and $\mathrm{X}=\mathrm{S}, \mathrm{Se})^{14-18}$ comprising a monolayer structure with $\mathrm{X}-\mathrm{M}-\mathrm{X}$ and layered III-VI compounds $\mathrm{NX}(\mathrm{N}=\mathrm{Ga}$, In and $\mathrm{X}=$ $\mathrm{S}$, Se, Te $)^{19-23}$ that are composed of fundamental units of $\mathrm{X}-\mathrm{N}-\mathrm{N}-\mathrm{X}$ might be two of the important braches of $2 \mathrm{D}$ materials that require further research and development in electronics and optoelectronics devices applications. The TMDC of $\mathrm{ReSe}_{2}$ has been proven to be a bipolar channel material for application in analog and digital integrated circuits ${ }^{24}$. The layered TMDCs of $\mathrm{WSe}_{2}$ and $\mathrm{Cr}$-doped $\mathrm{WSe}_{2}$ possess the flexibility on bandgap tuning and also stacking phase change from $2 \mathrm{H}$ to $1 \mathrm{~T}$ with the increase of the chromium content ${ }^{25}$. A plasma-treated $\mathrm{MoS}_{2}$ device was proposed to be a multibits memory that applied for improving computation speed and data storage capacity ${ }^{26}$. Conversely, considering III-VI compounds, InSe has been demonstrated as a high-mobility 2D semiconductor $\left(1006-3700 \mathrm{~cm}^{2} / \mathrm{V}\right.$-s) that is suitable for the fabrication of high-speed field-effect transistors (FETs) ${ }^{7,8}$. The native oxidation layer of $\operatorname{lnO} \mathrm{O}_{x}$ on an InSe layer could also produce artificial synapse functions in an InSe FET device ${ }^{27}$. The layered III-VI GaSe ${ }_{1-x} S_{x}(0 \leq x \leq 1)$ series compounds are optically sensitive and can be applied in photodetectors and light-emission devices in the visible-to-ultraviolet region ${ }^{28-30}$. Recently, as an additional class of $2 \mathrm{D}$ layered materials with a monolayer structure between that of TMDCs and III-VI compounds, metal phosphorus trichalcogenides (also known as metal phosphotrichalcogenides) $\mathrm{M}_{2} \mathrm{P}_{2} \mathrm{X}_{6}(\mathrm{M}=\mathrm{V}, \mathrm{Mn}, \mathrm{Fe}, \mathrm{Co}, \mathrm{Ni}, \mathrm{Cd}, \mathrm{Mg}$, or $\mathrm{Zn} ; \mathrm{X}=\mathrm{S}$ or Se) have attracted considerable attentions ${ }^{31,32}$. In the metal phosphotrichalcogenides $M_{2} P_{2} X_{6}$, each $P_{2} X_{6}$ unit has the valency of $4-$ [i.e., $\left(P_{2} X_{6}\right)^{4-}$ ]. It is necessary to have $M_{2}^{4+}$ ions for achieving the whole $\mathrm{M}_{2} \mathrm{P}_{2} \mathrm{X}_{6}$ structure, e.g., $\mathrm{Mg}_{2} \mathrm{P}_{2} \mathrm{~S}_{6}, \mathrm{Zn}_{2} \mathrm{P}_{2} \mathrm{Se}_{6}$, and $\mathrm{Ni}_{2} \mathrm{P}_{2} \mathrm{~S}_{6}$, etc ${ }^{31}$.
However, the $\mathrm{M}_{2}{ }^{4+}$ ions are not usually the same metal elements, thus some of the compounds like CulnP $\mathrm{S}_{6}, \mathrm{CuCrP}_{2} \mathrm{~S}_{6}$, and Agln $\mathrm{P}_{2} \mathrm{Se}_{6}$, etc. also belong to the metal phosphotrichalcogenide family $^{32}$. According to theoretical and experimental results, the bandgap values of $\mathrm{M}_{2} \mathrm{P}_{2} \mathrm{X}_{6}$ (as well as $\mathrm{MPS}_{3}$ ) range from 1.2 to $3.4 \mathrm{eV}^{32}$, which may considerably enhance the absorption efficiency and wavelength absorption region compared with those of layered TMDCs having bandgap values of 1.2 to $2 \mathrm{eV}$. These materials typically own chemical bonding that is intermediate between covalent and ionic as well as exhibit unusual behavior of intercalation-substitution ions, which can render them suitable for applications in Li-ion batteries ${ }^{33}$. Moreover, some of the metal elements in this family are usually magnetic (e.g., $\mathrm{M}=\mathrm{Fe}, \mathrm{Ni}, \mathrm{Mn}$ and $\mathrm{Co}$ ). As these metal ions are formed in the van der Waals sheet of $\mathrm{M}_{2} \mathrm{P}_{2} \mathrm{~S}_{6}$, each metal atom would have three equal-distance $M$ neighbors and exhibit stable magnetic phases in the hexagonal lattice. Therefore, layered $\mathrm{MPX}_{3}$ has been proposed to exhibit specific applications in magneto-optics ${ }^{34}$, magnetic storage, and 2D magnetism ${ }^{35}$. The Néel temperature $\left(T_{N}\right)$ is an index of the magnetic transition from antiferromagnetic to paramagnetic. The values of $T_{\mathrm{N}}$ are $\sim 78 \mathrm{~K}$ for $\mathrm{MnPS}_{3}, \sim 123 \mathrm{~K}$ for $\mathrm{FePS}_{3}$, and $\sim 155 \mathrm{~K}$ for layered $\mathrm{NiPS}_{3}$. NiPS 3 exhibits the highest Néel temperature, and the magnetization axis of the ordered states lies along the van der Waals plane, which differs from those of layered $\mathrm{MnPS}_{3}$ and $\mathrm{FePS}_{3}$ with out-of-plane axial magnetization $^{35}$.

Beyond the study of magnetic properties, few-layer semiconducting $\mathrm{NiPS}_{3}$ also has been fabricated as an ultraviolet photoconductive photodetector with a high detectivity of $1.22 \times$ $10^{12}$ Jones as determined via Jones testing with a 254-nm laser ${ }^{36}$. The heteroatom doping of carbon on the $\mathrm{NiPS}_{3}$ surface activates the photocatalytic activity of the basal plane and enhances the hydrogen evolution efficiency in the $\mathrm{KOH}$ solution ${ }^{37}$. The catalytic behavior of $\mathrm{NiPS}_{3}$ also can be exploited to perform water splitting $^{38}$. A resistive-type humidity sensor with a high sensing speed constructed using layered $\mathrm{NiPS}_{3}$ also has been proposed ${ }^{39}$, and an n-channel $\mathrm{NiPS}_{3}$ FET with an on/off ratio reaching $10^{3}$ to

${ }^{1}$ Graduate Institute of Applied Science and Technology, National Taiwan University of Science and Technology, Taipei 106, Taiwan. ${ }^{2}$ Graduate Institute of Electro-Optical Engineering and Department of Electronic and Computer Engineering, National Taiwan University of Science and Technology, Taipei 106, Taiwan. ${ }^{凶}$ email: chho@mail.ntust.edu.tw 
$10^{5}$ also has been reported ${ }^{40}$. Although a few application studies of layered semiconducting $\mathrm{NiPS}_{3}$ have been reported, the fundamental band-edge nature and optical properties of the nickel phosphotrichalcogenide are not well understood. This in turn limits the development of electronic and optoelectronic devices prepared using $\mathrm{NiPS}_{3}$. Some studies have claimed that the bandgap of $\mathrm{NiPS}_{3}$ is $1.6 \mathrm{eV}^{31,32,41}$; however, the experimental band-edge structure and excitonic transitions in layered $\mathrm{NiPS}_{3}$ have not been explored in detail.

In this paper, the band-edge excitons of few-layer $\mathrm{NiPS}_{3}$ are first observed by temperature-dependent microthermal-modulated reflectance $(\mu \mathrm{TR})$ measurements in the temperature range between 10 and $300 \mathrm{~K}$. Owing to the reduced dimensionality serving to weaken the dielectric screening effect of electron-hole pairs, 2D materials usually have strong Coulumbic interactions in excitons and trions, among others, to form a many-body system, thereby achieving increased excitonic (trionic) binding energy in the layer plane ${ }^{42}$. Excitonic transitions are thus frequently constructed and exist in 2D layered semiconductors. The most renowned cases of this include the $A$ and $B$ excitons that are present in layered $\mathrm{MoS}_{2}{ }^{12}$, which are also clearly present in the entire series of $\mathrm{Mo}_{1-x} \mathrm{~W}_{\mathrm{x}} \mathrm{S}_{2}(0 \leq x \leq 1)$ layers with an energy blueshift behavior with the increase in the $W$ content $^{43}$. Another typical case is the five dipole excitons of $E_{1}{ }^{\mathrm{ex}}, E_{2}{ }^{\mathrm{ex}}, E_{3}{ }^{\mathrm{ex}}, E_{\mathrm{S} 1}{ }^{\mathrm{ex}}$, and $E_{S 2}{ }^{\text {ex }}$, respectively, with a mutual orthogonality of polarization in the layered 2D TMDCs of $\operatorname{ReS}_{2}$ and $\mathrm{ReSe}_{2}{ }^{44-46}$. In this study, prominent and clear excitonic transitions of few-layer $\mathrm{NiPS}_{3}$ $(t=15 \pm 5 \mathrm{~nm})$ are observed by $\mu \mathrm{TR}$ measurements at $10 \mathrm{~K}$ near the band edge. The band-edge excitons of $\mathrm{NiPS}_{3}$ contain three excitonic features denoted as $A_{1}, A_{2}$, and $E_{\infty}$ in the $A$ series excitons, respectively, while a broadened feature correlates with the band-to-band feature of $\mathrm{B}$. The $A_{1}, A_{2}$, and $E_{\infty} \mu \mathrm{TR}$ features display the Rydberg-series-like transitions with the first level at $A_{1}$, the second level at $A_{2}$, and the continuum band at $E_{\infty}$, respectively. The $B \mu T R$ feature may originate from valence-band $\left(E_{\mathrm{V}}\right)$ splitting to the direct conduction-band $\left(E_{\mathrm{C}}\right)$ edge transition. The temperature dependences of the energies and broadening parameters of the A series and B obtained via $\mu$ TR measurements of few-layer $\mathrm{NiPS}_{3}$ also are analyzed. In addition, temperaturedependent transmittance (optical absorption) measurements of layered $\mathrm{NiPS}_{3}$ are conducted to verify and identify the absorption edge and excitonic transitions of the A series from 10 to $300 \mathrm{~K}$. The direct optical gap $E_{\infty}$ of $\mathrm{NiPS}_{3}$ is determined to be around $1.402 \mathrm{eV}$ at $300 \mathrm{~K}$. According to thickness-dependent micro-photoluminescence ( $\mu \mathrm{PL})$ measurements, an indirect-like $E_{3 \mathrm{~d}}$ emission peak that merged with $A_{1}$ is detected at $\sim 1.23 \mathrm{eV}$, while a direct emission of $\mathrm{B}$ band also appears at $\sim 1.825 \mathrm{eV}$ at $300 \mathrm{~K}$ (i.e., $t=10-200 \mathrm{~nm}$ ). With changing the layer thickness of $\mathrm{NiPS}_{3}$, the PL intensities of the $E_{3 d}$ emission are also changed, while the intensity of the Bband emission is unchanged from $t=10-200 \mathrm{~nm}$. The Ni $3 d e_{\mathrm{g}}{ }^{*}$ band may be located intermediate between $\mathrm{E}_{\mathrm{C}}$ of $\mathrm{S} 3 p^{*}+\mathrm{P} 3 p^{*}+$ $\mathrm{Ni} 3 d^{*}$ and $\mathrm{E}_{V}$ of $\mathrm{Ni} 3 d+\mathrm{S} 3 p+\mathrm{P} 3 p$ to result in the $\mathrm{E}_{3 d}$ indirectlike emission in the layered $\mathrm{NiPS}_{3}$.

\section{RESULTS}

Crystal information and structure

Layered single crystals of nickel phosphotrisulfide $\mathrm{NiPS}_{3}$ were grown by the chemical vapor transport (CVT) method using $I_{2}$ as the transport agent. Supplementary Fig. 1a in the supplementary information (SI) shows the crystal morphology of the as-grown $\mathrm{NiPS}_{3}$ layered crystals for illustration. The outline of the layered $\mathrm{NiPS}_{3}$ single crystals essentially reveals a hexagonal shape, and the powdered X-ray diffraction (XRD) pattern shown in Supplementary Fig. $1 \mathrm{~b}$ exhibits a monoclinic structure (symmetry $\mathrm{C} 2 / \mathrm{m}$ ), with calculated lattice constants of $a=5.761, b=10.06, c=6.576 \AA$, and $\beta=107^{\circ}$. These values are in agreement with results obtained previously for $\mathrm{NiPS}_{3}{ }^{32,47,48}$. Supplementary Figs. 2-4 in the SI also include content analysis conducted by energy-dispersive X-ray analysis and structural characterization using Raman spectroscopy for comparison (see Supplementary Notes 1-2).

The A-series excitons and $\mathrm{B}$ band transition in $\mathrm{NiPS}_{3}$ at $10 \mathrm{~K}$

Figure 1a shows the low-temperature $\mu$ TR spectrum of a few-layer $\mathrm{NiPS}_{3}$ sample (i.e., black solid line) measured at $10 \mathrm{~K}$. The upper inset shows a microscope image of the few-layer $\mathrm{NiPS}_{3}$ nanoflake exfoliated on a $\mathrm{SiO}_{2} / \mathrm{Si}$ substrate for the $\mu \mathrm{TR}$ experiment, while the lower inset shows the thickness information determined by atomic force microscopy (AFM). The thickness is about 23 monolayers $\left(t=15 \pm 5 \mathrm{~nm}\right.$ ) of the layered $\mathrm{NiPS}_{3}$ crystal. As shown in Fig. 1a, the black solid line is the experimental spectrum, and the green-dashed curve is the least-square fit to a first-derivative Lorentzian line-shape function appropriate for the excitonic transitions expressed as follows ${ }^{49}$ :

$$
\frac{\Delta R}{R}=\operatorname{Re}\left[\sum_{i=1}^{n} A_{\mathrm{i}}^{\mathrm{ex}} e^{-j \phi_{\mathrm{i}}^{\mathrm{ex}}}\left(E-E_{\mathrm{i}}^{\mathrm{ex}}+j \Gamma_{\mathrm{i}}^{\mathrm{ex}}\right)^{-2}\right],
$$

where $i$ is the respective transition; $A_{i}^{\text {ex }}$ and $\phi_{i}^{\text {ex }}$ are the amplitude and phase of the line shape; and $E_{\mathrm{i}}^{\mathrm{ex}}$ and $\Gamma_{\mathrm{i}}^{\mathrm{ex}}$ are the energy and broadening parameter of the interband transitions, respectively. As analyzed by the Lorentzian line-shape fitting using Eq. (1), the energy values of two prominent excitonic features (i.e., $A_{1}=$ $1.475 \mathrm{eV}$ and $A_{2}=1.495 \mathrm{eV}$ ) together with one higher-energy transition (i.e, $E_{\infty}$ at $1.511 \mathrm{eV}$ ) are clearly detected in the $A$ series of few-layer $\mathrm{NiPS}_{3}$ by $\mu \mathrm{TR}$. One broadened $\mu \mathrm{TR}$ feature (denoted as B at $\sim 1.894 \mathrm{eV}$ ) is also found at the higher-energy side of $\mathrm{NiPS}_{3}$ at $10 \mathrm{~K}$. The transition amplitudes $A_{i}^{\mathrm{ex}}$ of the $\mathrm{A}$ series excitons gradually decrease from the maximum $n=1$ level, progressing to the subsequent $n=2$ transition, and finally the $E_{\infty}$ feature follows the general transition probability of the exciton sequence in semiconductors. Modulated TR measurements of semiconductors have been proven to be effective for the characterization of excitons, direct band edge, and interband transitions in the semiconductor's band structure ${ }^{50-52}$. The derivative spectral line features suppress the unintentional spectral background and emphasize the direct critical-point transitions in the band structure ${ }^{52}$. The TR modulation spectroscopy can be regarded as a physical derivative approach to the reflectance spectrum of semiconductor dielectric function by directly applying thermal perturbation to the crystal lattice periodically (i.e., $\Delta R$ ). The wellderivative $\Delta R$ signal (AC) of sample is thus measured and normalized to the sample reflectance $\mathrm{R}$ (DC) for obtaining $\Delta R / R$ at each wavelength using lock-in amplifier. The TR modulation spectroscopy is different from that of reflectance contrast method with firstly measuring $\Delta R / R=\left(R_{\text {sample }}-R_{\text {substrate }}\right) / R_{\text {substrate }}{ }^{53,54}$ and then implementing mathematical derivative to the line shape using $(\mathrm{d} / \mathrm{d} E)(\Delta R / R)^{53}$. The $\mu \mathrm{TR}$ technique is directly response to the physical derivative nature of direct transitions in the band structure of the few-layer sample, and thus possesses less substrate effect coming from the $\mathrm{SiO}_{2} / \mathrm{Si}$ substrate. Supplementary Fig. 5 shows the bulk TR results of $\mathrm{NiPS}_{3}$ without any substrate. Essentially the results measured by $\mu \mathrm{TR}$ (with substrate) and measured by conventional TR (without substrate) are similar (Supplementary Note 3). As shown in Fig. 1a, the sharp features of the $A$ series excitons and the extremely broadened $B$ transition reveal that the $A$ and $B$ excitons exhibit different origins in $\mathrm{NiPS}_{3}$. The crystal structure of $\mathrm{NiPS}_{3}$ can be realized as a stacking structure of one-layer trigonal $(1 \mathrm{~T})$ phase TMDCs such as $\mathrm{TiS}_{2}$. Figure $1 \mathrm{~b}$ shows the atomic arrangements of the side (upper parts) and top (lower part) views of the layered $\mathrm{NiPS}_{3}$ structure. As the $1 \mathrm{~T}-\mathrm{TiS}_{2}$ stacking formula, the $\mathrm{NiPS}_{3}$ phase is taken as the layered $\mathrm{MS}_{2}$ (as TMDCs) with the Ti atom replaced by $\mathrm{Ni}$, while $1 / 3$ of the $\mathrm{Ni}$ atoms are substituted by the $\mathrm{P}-\mathrm{P}$ pairs ( $\mathrm{P}_{2}$ dimers) to form $\mathrm{P}_{2} \mathrm{~S}_{2}+\mathrm{NiS}_{2}+\mathrm{NiS}_{2}=\mathrm{Ni}_{2} \mathrm{P}_{2} \mathrm{~S}_{6}$ structures that connect and 


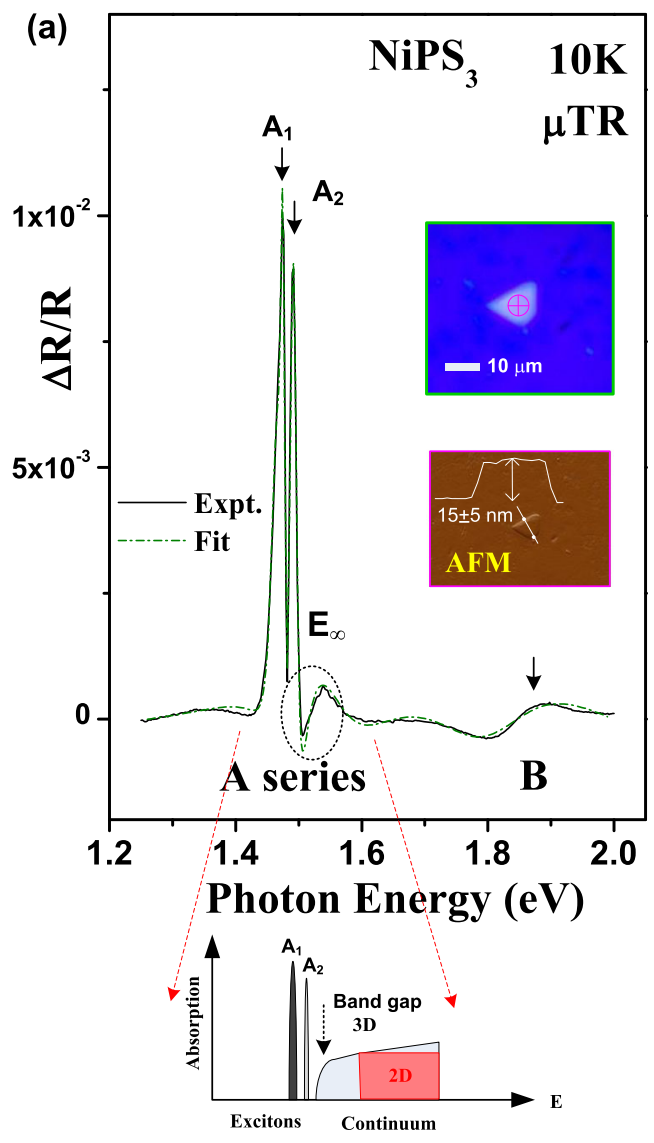

(b) $\mathrm{Ni}_{2} \mathrm{P}_{2} \mathrm{~S}_{6}$ structure, side view and top view

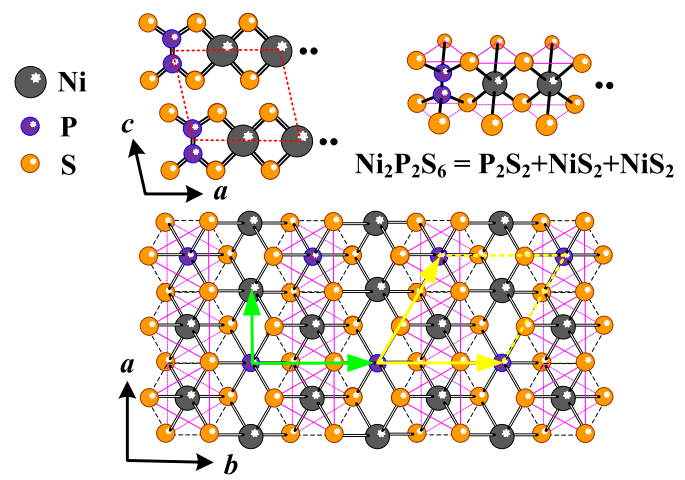

(c)

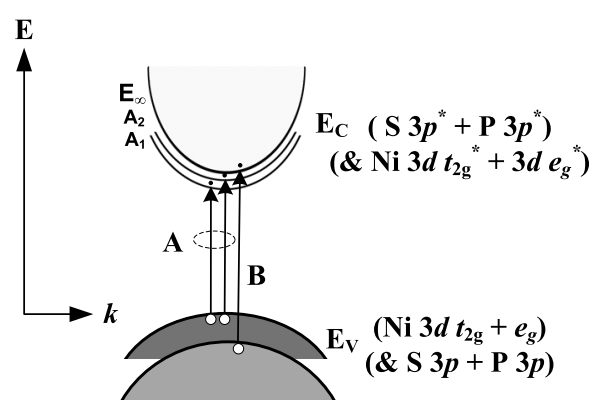

Fig. 1 Low-temperature excitonic series observed in NiPS $_{\mathbf{3}}$. a Band-edge excitons of the A series and B transition in few-layer NiPS 3 observed via $\mu \mathrm{TR}$ measurements at $10 \mathrm{~K}$. Insets show the microscopic image and AFM results of the few-layer sample on an $\mathrm{SiO}_{2} / \mathrm{Si}$ substrate. The lower inset depicts the representative scheme of Rydberg series absorption for contrast. $\mathbf{b}$ Atomic arrangements of the side and top views for the layered $\mathrm{Ni}_{2} \mathrm{P}_{2} \mathrm{~S}_{6}$ structure. c Representative band-edge scheme for the A series exciton and $\mathrm{B}$ transition in NiPS ${ }_{3}$.

extend to the entire lamella sheet (monolayer). This situation is clearly demonstrated in the side view of the monolayer $\mathrm{NiPS}_{3}$ that is displayed in the upper parts of Fig. 1b. Considering the bonding structure of $\mathrm{NiPS}_{3}$, the P-P pairs are covalently bonded to six sulfur atoms, and each $\mathrm{P}$ atom is tetrahedrally coordinated with three $\mathrm{S}$ atoms to form opposite triangles, as shown by the $1 \mathrm{~T}$ phase shown in the top view of Fig. 1b. Therefore, a $\left(\mathrm{P}_{2} \mathrm{~S}_{6}\right)^{4-}$ fundamental unit exists, and it requires two $\mathrm{Ni}^{2+}$ to construct the complete $\mathrm{Ni}_{2} \mathrm{P}_{2} \mathrm{~S}_{6}$ layer compound. Because the $\mathrm{P}_{2} \mathrm{~S}_{2}$ is occupied in the $1 / 3$ lattice of layered $\mathrm{Ni}_{2} \mathrm{P}_{2} \mathrm{~S}_{6}$, the perfect hexagonal $1 \mathrm{~T}$ phase is thereby transformed into a monoclinic layered structure of $\mathrm{C} 2 / \mathrm{m}$ symmetry. The $\mathrm{P}_{2}$ dimers also may contribute to the band-edge electronic states of layered $\mathrm{NiPS}_{3}$. Therefore, the antibonding states of $\mathrm{S} 3 p^{*}+\mathrm{P} 3 p^{*}+\mathrm{Ni} 3 d^{*}$ may consist in conduction band while the valence band is composed by hybridation of $\mathrm{Ni} 3 d+\mathrm{S} 3 p+\mathrm{P} 3 p$ bonding states. Excepting the incorporation of the $\mathrm{P}-\mathrm{P}$ pairs, the band-edge contributions of electronic states in $E_{\mathrm{C}}$ and $E_{\mathrm{V}}$ of $\mathrm{NiPS}_{3}$ can also refer to the TMDCs as $\mathrm{MoS}_{2}{ }^{55}$, where the largely Mo $4 d^{*}$ and minor $S 3 p^{*}$ are simultaneously consisted in the $E_{\mathrm{C}}$ bottom while major Mo $4 d$ and $S 3 p$ are positioned at the top of $E_{\mathrm{V}}$. For the spin-orbit splitting band $\Delta_{\mathrm{so}}$, the band contributions may consisted of $60 \%$ Mo $4 d_{\mathrm{z}}^{2}$ and $40 \% \mathrm{~S} 3 p_{\mathrm{z}}{ }^{55}$. The main difference between the band-edge structures of $\mathrm{MoS}_{2}$ and $\mathrm{NiPS}_{3}$ is maybe the magnetic structure of $\mathrm{Ni} 3 d$ states that contributed to the antiferromagnet layered $\mathrm{NiPS}_{3}$.

According to the analysis of exciton series A observed in Fig. $1 \mathrm{a}$, a modified hydrogen Rydberg model of $2 D^{53}$ derived from $3 D$ case $^{56}$ may be used to analyze optical transitions and to evaluate binding energy of the observed excitons. In the 2D case, the model employs an effective Hamitonian $H=-\hbar^{2} \cdot \nabla_{\mathrm{r}}^{2} / \mu+V_{\mathrm{e}-\mathrm{h}}(r)$, where $\mu^{-1}=m_{\mathrm{e}}^{-1}+m_{\mathrm{h}}^{-1}$ ( $\mu$ : reduced mass) and $V_{\mathrm{e}-\mathrm{h}}(r)=-e^{2} /\left(\varepsilon_{\mathrm{eff}} \cdot r\right)$ is the locally attractive Columbic interaction between electron and hole. The term $\varepsilon_{\text {eff }}$ is the effective permittivity of the material available for excitons. The 2D model of exciton predicts an excitonic binding energy expressed as follows:

$E_{\mathrm{b}}^{\mathrm{n}}=\mu \cdot e^{4} /\left[2 \hbar^{2} \cdot \varepsilon_{\text {eff }}^{2} \cdot(n-1 / 2)^{2}\right]=E_{\infty}-E_{\mathrm{An}}$,

where $n$ is the principal quantum number, $E_{\infty}$ is the threshold energy of the excitonic sequence, and $E_{A n}$ is the transition energy of the exciton level detected by the layered sample. According this 2D model, a typical case for estimating $A$ series excitons in monolayer $(1 \mathrm{~L}) \mathrm{WS}_{2}$ is $E_{\infty}=2.41( \pm 0.04) \mathrm{eV}$ and binding energy $E_{\mathrm{b}}{ }^{1}=0.32( \pm 0.04) \mathrm{eV}$. However, for the thick-layer bulk case $(>10 \mathrm{~L})$, because the effects caused by 3D hydrogenic Hamitonian and excitonic Bohr radius, etc. the direct gap reduces to $E_{\infty}=2.10 \mathrm{eV}$ and $E_{\mathrm{b}}{ }^{1}$ decreases to $0.05 \mathrm{eV}^{53}$. For the $n=2$ level, the energy separation between $n=1$ and $n=2$ is increased with the layer thickness decreases when $t \leq 4 \mathrm{~L}$. The energy splitting of $n=1$ and $n=2$ will remain the same in bulk case in layered $\mathrm{WS}_{2}(t>10 \mathrm{~L})^{57}$. For $\mathrm{NiPS}_{3}$, the $\mathrm{A}$ series and $\mathrm{B}$ transitions are correlated with majorly $\mathrm{Ni} 3 d$ states constructed in $E_{\mathrm{C}}$ and $E_{\mathrm{V}}{ }^{58-61}$. The $A$ series originates from largely $d$-to- $d$ transitions correlated with the spin orientation in the antiferromagnetic $\mathrm{NiPS}_{3}{ }^{58}$. As shown in Fig. 1a, the $A_{1}$ level is tentatively assigned to be the $n=1$ level, the $A_{2}$ exciton is inferred to be the $n=2$ level, and $E_{\infty}$ feature is correlated with the direct band gap of $\mathrm{NiPS}_{3}$. The lower inset below Fig. 1a depicts the representative scheme of the $A_{1}, A_{2}$, and continuum band of bulk 
$\mathrm{NiPS}_{3}$ for analog to the detected $\mathrm{A}$ series excitons at $10 \mathrm{~K}$. The $A_{1}$ exciton of $\mathrm{NiPS}_{3}$ has also recently been detected by $\mathrm{PL}$ measurement to locate between 1.475 and $1.478 \mathrm{eV}$ with the thickness changing from $3 \mathrm{~L}$ to $8 \mathrm{~L}$ at $10 \mathrm{~K}^{58}$. For the bilayer and monolayer $\mathrm{NiPS}_{3}$, the PL emission of the $A_{1}$ exciton is missing to lend evidence that monolayer $\mathrm{NiPS}_{3}$ is maybe an indirect-like 2D material. The excitonic PL emission of $A_{1}$ also showed linearlypolarized light along the crystal's $a$ axis ${ }^{58}$. The polarized behavior of the $A_{1}$ exciton is different from the $\mathrm{A}$ series exciton in the layered $\operatorname{MoS}_{2}{ }^{12}$. The $A_{1}$ exciton had been assigned as a Zhang-Rice triplet to a Zhang-Rice singlet transition that correlated with the spin directions in the $\mathrm{Ni} 3 d$ states of $\mathrm{NiPS}_{3}{ }^{58}$. Figure 1c shows the representative band scheme of $\mathrm{NiPS}_{3}$ appropriate for the transitions of the $A$ series and $B$ observed in Fig. 1a by $\mu T R$. The band block array of $\mathrm{NiPS}_{3}$ is proposed to be the $\mathrm{S} 3 p^{*}+\mathrm{P} 3 p^{*}$ antibonding states combined with the lower $\mathrm{Ni} 3 d^{*}$ band that is positioned at the bottom of $\mathrm{E}_{\mathrm{C}}$, whereas the fully occupied $\mathrm{Ni} 3 d$ $\left(t_{2 \mathrm{~g}}+e_{\mathrm{g}}\right)$ followed by the $\mathrm{S} 3 p+\mathrm{P} 3 p$ bonding state comprises the main $E_{\mathrm{V}}^{57}$. The Ni $3 d$ electron state is $d^{8}$, which separates into the lower fully-occupied $t_{2 \mathrm{~g}}{ }^{6}$ in $E_{\mathrm{V}}$ (i.e., $E_{\mathrm{V}}$ is mainly Ni $3 d t_{2 \mathrm{~g}}{ }^{6}+\mathrm{P} 3 p+\mathrm{S}$ $3 p$ ) and the higher half-filled $e_{\mathrm{g}}{ }^{2}$ band hybridization with $E_{\mathrm{C}}$ (i.e., $E_{\mathrm{C}}$ is major in $\left.\mathrm{Ni} 3 d^{*} e_{\mathrm{g}}{ }^{2}+\mathrm{P} 3 p^{*}+\mathrm{S} 3 p^{*}\right)$, where $\mathrm{P} 3 p_{\mathrm{z}}^{*}$ antibonding state contributes to the $E_{\mathrm{C}}$ of $\mathrm{NiPS}_{3}{ }^{59,60}$. Not only the Ni $3 d$ state but also the $\mathrm{P} 3 p$ state hybridizes with the $\mathrm{S} 3 p$ orbital still contributing to the band edge of layered $\mathrm{NiPS}_{3}{ }^{61}$. As shown in Fig. $1 \mathrm{C}$, the $\mathrm{A}$ series excitons of the $A_{1}, A_{2}$, and $E_{\infty}$ transitions may originate from the top of $E_{\mathrm{V}}$ by Ni $3 d$ to the $E_{\mathrm{C}}$ bottom of $\mathrm{NiPS}_{3}$, while the $B$ feature is inferred to originate from the spin-orbital splitting of $E_{V}$ to the $E_{C}$ bottom. The transition of spin-orbital splitting band to $E_{C}$ is rather flat to obtain a more broadened $\mathrm{B}$ feature as compared to the sharp features of the A series excitons in Fig. 1a.

Temperature-dependent microthermal-modulated reflectance spectroscopy of few-layer $\mathrm{NiPS}_{3}$

Figure $2 \mathrm{a}, \mathrm{b}$ shows the temperature-dependent $\mu \mathrm{TR}$ spectra of the few-layer $\mathrm{NiPS}_{3}$ sample in the temperature range between 10 and $300 \mathrm{~K}$ near the band edge. The dashed lines are the experimental spectra, and the solid curves are the least-square fits using Eq. (1). The obtained transition energies $E_{\mathrm{i}}^{\mathrm{ex}}$ of $A_{1}, A_{2}, E_{\infty}$, and $B$ transitions from 10 to $300 \mathrm{~K}$ are depicted in Fig. $3 \mathrm{a}$ and temperaturedependent broadening parameters $\Gamma_{i}^{\mathrm{ex}}$ of the $A_{1}$ and $B \mu \mathrm{TR}$ features are shown in Fig. 3b, respectively. The obtained transition energies of $A_{1}, A_{2}, E_{\infty}$, and $B$ at $10 \mathrm{~K}$ are indicated by arrows in

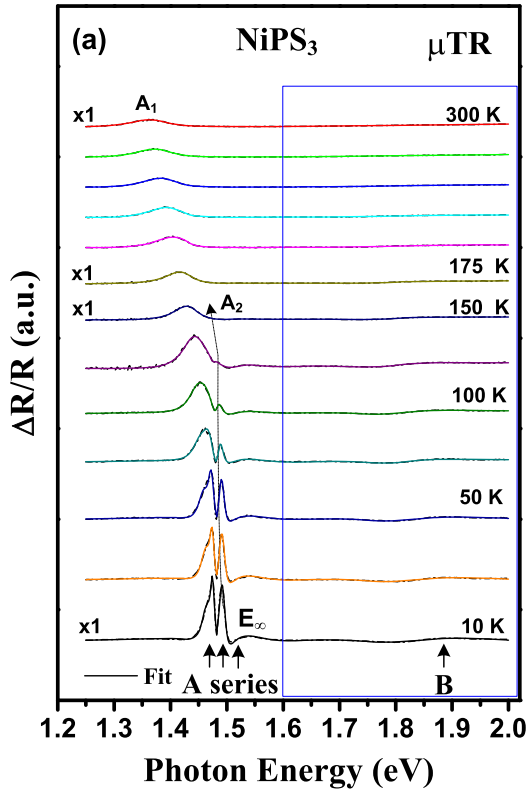

(c)

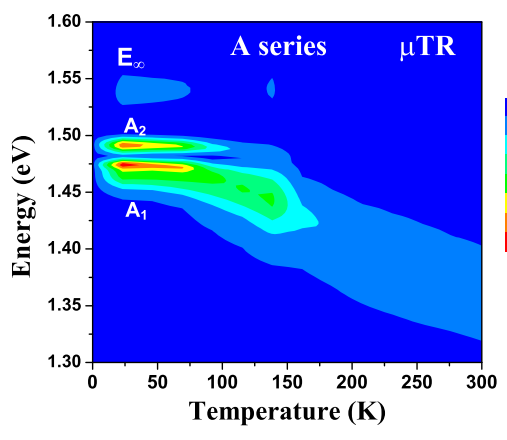

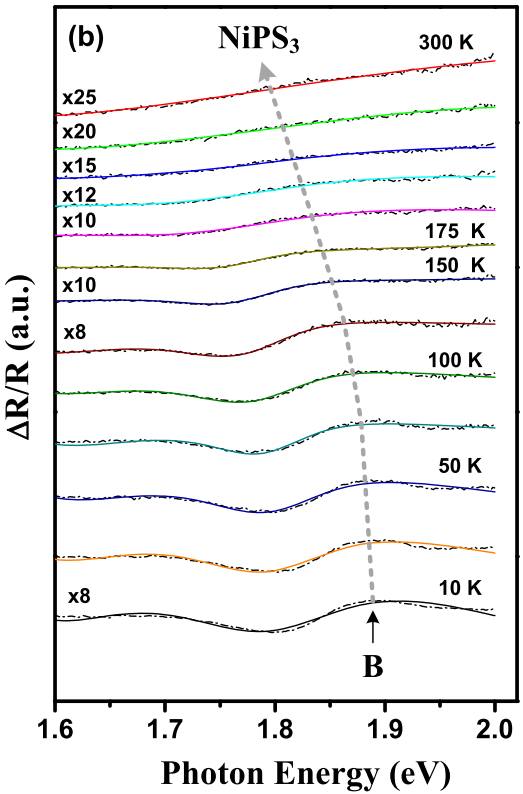

(d)

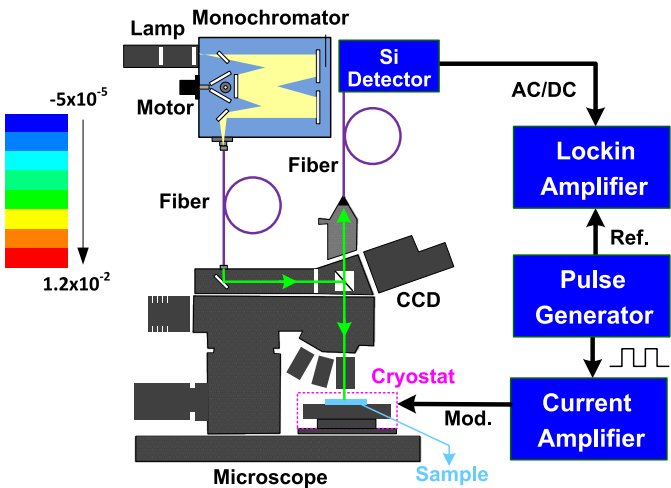

Fig. 2 Temperature-dependent $\mu$ TR spectra of $\mathrm{NiPS}_{3}$ near band edge. a Temperature dependence of the band-edge excitonic transitions of the $A$ series and $B$ observed in few-layer $\mathrm{NiPS}_{3}$ via $\mu \mathrm{TR}$ measurements. The solid lines are the least-square fits of the experimental data to a derivative of the Lorentzian line-shape function in Eq. (1). b Magnification of the B transition feature in a. c 2D contour plot of the $\mu$ TR spectra of the A series excitons from 10 to $300 \mathrm{~K}$ to illustrate the temperature-energy shift and intensity change. d Representative scheme of the experimental setup for the temperature-dependent $\mu$ TR measurements. 

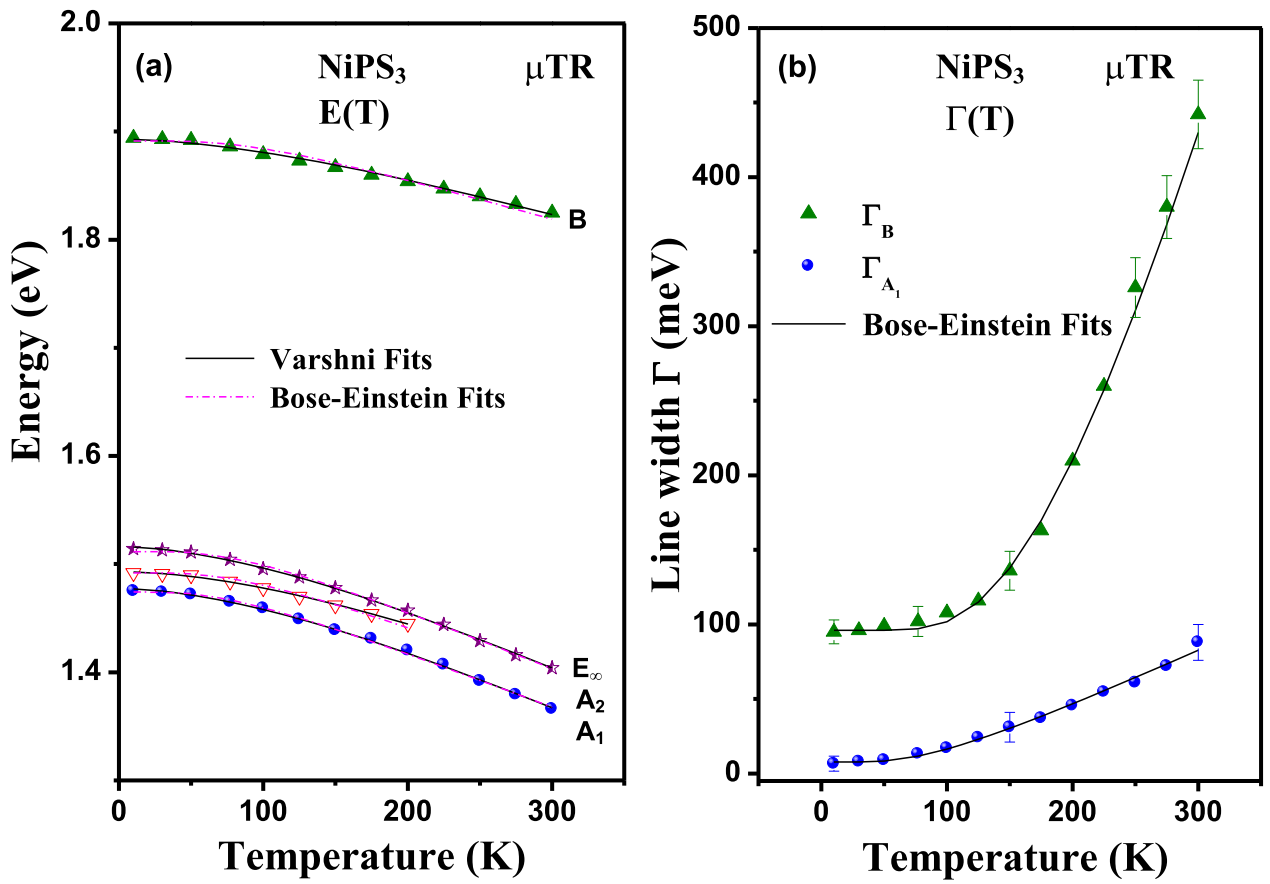

Fig. 3 Temperature dependence energies and broadening parameters of the A series and B features obtained by $\mu$ TR. a Temperaturedependent transition energies of the $A$ series and B features obtained by $\mu$ TR measurements. The symbols are the data points, solid lines are the least-square fits of the Varshni type, and dashed lines are those of the Bose-Einstein fitting. $\mathbf{b}$ Temperature dependence of the line-width broadening parameter of the $A_{1}$ and $B$ features obtained by $\mu$ TR experimentation.

Fig. $2 a$, b. The energy values are $A_{1}=1.475 \mathrm{eV}, A_{2}=1.495 \mathrm{eV}, E_{\infty}=$ $1.511 \mathrm{eV}$, and $B=1.894 \mathrm{eV}$, respectively. As shown in Fig. 2a, with the increase in the temperature from 10 to $300 \mathrm{~K}$ in the few-layer $\mathrm{NiPS}_{3}$, band-edge excitons of the $\mathrm{A}$ series reveal energy reduction and line-shape broadened characteristics. Figure $2 \mathrm{~b}$ shows the magnification of the $\mu$ TR features of the B exciton from 1.6 to $2 \mathrm{eV}$ (blue box in Fig. 2a): Energy reduction and line-shape broadened characteristics are clearly observed from 10 to $300 \mathrm{~K}$. The transition amplitudes $A_{i}^{\text {ex }}$ of the $A$ series and $B$ features in Fig. $2 \mathrm{a}, \mathrm{b}$ also demonstrate degradation with the increase in the temperature. Figure $2 \mathrm{c}$ shows the $2 \mathrm{D}$ contour plot of the $\mu \mathrm{TR}$ spectra of the A series excitons, $A_{1}, A_{2}$, and $E_{\infty}$ from 10 to $300 \mathrm{~K}$. The energy separation of the $n=1$ and $n=2$ levels of $\mathrm{NiPS}_{3}$ is about $20 \mathrm{meV}$ (Fig. 2(a)). The $n=2$ exciton level is ionized at $T>$ $175 \mathrm{~K}$ (Fig. 2a, c). The value of the thermal energy $k T$ (where $k$ is the Boltzmann constant) is in agreement with the energy separation of the $n=2$ level and $E_{\infty}(\sim 16 \mathrm{meV})$ to facilitate the thermal ionization of the $A_{2}$ exciton level. The binding energy of the $A_{1}$ level obtained from the Rydberg-series analysis of $\mathrm{NiPS}_{3}$ in Eq. (1) is about $36 \mathrm{meV}$. Therefore, the $A_{1}$ exciton (i.e., $A_{1} \cong$ $1.366 \mathrm{eV}$ ) can be detected in Fig. 2a for the layered $\mathrm{NiPS}_{3}$ at $300 \mathrm{~K}$. Figure $2 d$ shows the representative scheme for the experimental setup of the $\mu \mathrm{TR}$ measurement for the few-layer $\mathrm{NiPS}_{3}$. A tungsten halogen lamp dispersed by a monochromator provided the monochromatic light, and which was then guided to a CCDequipped microscope using optical fiber. The detailed descriptions of the experimental setup are stated in "Methods" section.

Temperature-dependent analysis of $A$ series and $B$ in $\mathrm{NiPS}_{3}$ Figure $3 a$ shows the experimental data points of the transition energies of the temperature-dependent $\mathrm{A}$ series $(n=1, n=2$, and $E_{\infty}$ ) and $\mathrm{B}$ features in the few-layer $\mathrm{NiPS}_{3}$ obtained by the analysis of the derivative Lorentzian line-shape fitting using Eq. (1) that was derived from Fig. 2a, b. The solid lines correspond to the least-square fits to the Varshni empirical relationship:

$$
E_{\mathrm{i}}(T)=E_{\mathrm{i}}(0)-a_{\mathrm{i}} \cdot T^{2} /\left(\beta_{\mathrm{i}}+T\right),
$$

where $i$ is the respective transition, $E_{\mathrm{i}}(0)$ is the energy at $0 \mathrm{~K}, a_{\mathrm{i}}$ is related to the strength of the electron (exciton)-phonon interaction, and $\beta_{\mathrm{i}}$ is closely related to the Debye temperature. Table 1 lists the obtained fitting parameters for comparison. More rapid temperature-energy shift of the $E_{\mathrm{V}}$ top than that of the spin-orbital splitting in the $\mathrm{NiPS}_{3}$ band indicates that the exciton-phonon interaction strength of the A-series are slightly larger than that of the $B$ band transition. According to the energy difference between $B$ and $E_{\infty}$ in Fig. 3a, the spin-orbital splitting $\left(\Delta_{\text {so }}\right)$ of the few-layer $\mathrm{NiPS}_{3}$ is about $0.383 \mathrm{eV}$. This value is greater than that of about $0.19 \mathrm{eV}$ in the energy separation of the $A$ and $B$ excitons in $\mathrm{MoS}_{2}{ }^{62,63}$. The temperature dependence of the bandedge transition energies of few-layer $\mathrm{NiPS}_{3}$ also can be analyzed using another expression containing the Bose-Einstein occupation factor for phonons: ${ }^{64}$

$$
E_{\mathrm{i}}(T)=E_{\mathrm{iB}}(0)-a_{\mathrm{iB}} \cdot\left\{1+2 /\left[\exp \left(\theta_{\mathrm{iB}} / T\right)-1\right]\right\},
$$

where $a_{\mathrm{iB}}$ represents the strength of the electron (exciton)-phonon interaction, and $\theta_{\mathrm{iB}}$ corresponds to the averaged phonon temperature. Figure $3 a$ shows the fitted results represented by the red dashed-doted curves. The obtained values of $E_{\mathrm{iB}}(0), a_{\mathrm{iB}}$, and $\theta_{\mathrm{iB}}$ also are listed in Table 1 together with the layered compounds $\mathrm{MoS}_{2}, \mathrm{WS}_{2}$, and $\mathrm{Mo}_{0.5} \mathrm{~W}_{0.5} \mathrm{~S}_{2}{ }^{43}$, while $\mathrm{GaAs}{ }^{64}$ and $\mathrm{ZnSe}^{65}$ are included for comparison. The values of the Varshni parameter $a_{\mathrm{i}}$ and Bose-Einstein type constant $a_{\mathrm{iB}}$ of the A series excitons $\left(A_{1}, A_{2}\right.$, and $\left.E_{\infty}\right)$ in $\mathrm{NiPS}_{3}$ are greater than those of the layered TMDCs $\mathrm{MoS}_{2}, \mathrm{Mo}_{0.5} \mathrm{~W}_{0.5} \mathrm{~S}_{2}$, and WS . The energy difference of the $A$ exciton between 10 and $300 \mathrm{~K}$ is about $110 \mathrm{meV}$ in $\mathrm{NiPS}_{3}$, which is greater than those of $70-80 \mathrm{meV}$ for $\mathrm{MoS}_{2}, \mathrm{Mo}_{0.5} \mathrm{~W}_{0.5} \mathrm{~S}_{2}$, and $\mathrm{WS}_{2}$. The incorporation of the additional $\mathrm{P}_{2}$ pairs in layered $\mathrm{Ni}_{2} \mathrm{P}_{2} \mathrm{~S}_{6}$ is potentially the main reason for causing a more rapid temperature-energy gap shift compared to the other layered TMDCs in the environment of a crystal lattice-constant change. 
Table 1. Values of the Varshni and Bose-Einstein type fitting parameters that describe the temperature dependences of the excitonic transition energies of the $A_{1}, A_{2}, E_{\infty}$, and $B$ in the few-layer NiPS $S_{3}$ together with those previously obtained for $\operatorname{MoS}_{2}, \mathrm{WS}_{2}, \mathrm{Mo}_{0.5} \mathrm{~W}_{0.5} \mathrm{~S}_{2}, \mathrm{GaAs}_{\mathrm{A}}$, and $\mathrm{ZnSe}$.

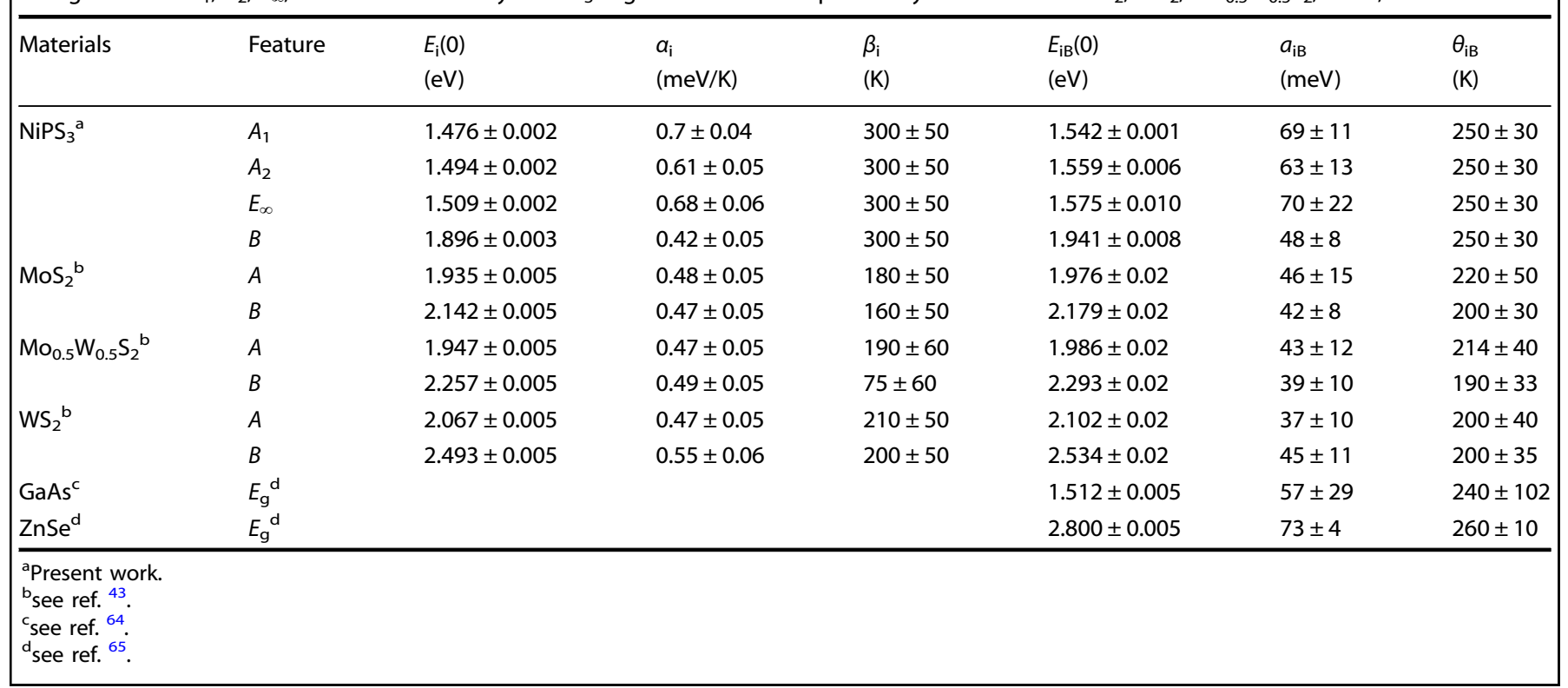

Figure $3 \mathrm{~b}$ plots the analysis of the broadening parameter $\Gamma$ (halfwidth at half-maximum (HWHM)) of the representative $A_{1}$ and $\mathrm{B}$ features in the $\mu \mathrm{TR}$ spectra of few-layer $\mathrm{NiPS}_{3}$ between 10 and $300 \mathrm{~K}$ with representative error bars. The solid lines represent the least-square fitting to a Bose-Einstein-type formula, which is appropriate for the temperature-dependent line-width analysis as follows: ${ }^{64,65}$

$\Gamma_{\mathrm{i}}(T)=\Gamma_{\mathrm{i} 0}+\Gamma_{\mathrm{iLO}} /\left[\exp \left(\theta_{\mathrm{iLO}} / T\right)-1\right]$

where $\Gamma_{i 0}$ represents the term of the line-shape broadening invoked from the mechanism of crystallinity from impurities, dislocations, electron interactions, and Auger processes, whereas the $\Gamma_{\text {iLO }}$ term is related to the electron (exciton)-longitudinal optical (LO) phonon (Frohlich) interaction. $\theta_{\mathrm{iLO}}$ is related to the LO phonon temperature. The obtained fitting parameters of the line-shape broadening of the $A_{1}$ and $\mathrm{B}$ transitions in $\mathrm{NiPS}_{3}$ are $\Gamma_{\mathrm{iO}}=7 \pm 1$ and $96 \pm 10 \mathrm{meV} ; \Gamma_{\mathrm{iLO}}=98 \pm 10$ and $1800 \pm 800 \mathrm{meV}$; and $\theta_{\mathrm{iLO}}=280 \pm 20$ and $584 \pm 140 \mathrm{~K}$, respectively. The considerably lower value of $\Gamma_{i 0}$ of the $A_{1}$ exciton indicates that an extremely high-quality crystal is obtained for layered $\mathrm{NiPS}_{3}$. The broadened $B$ feature implies that the spin-orbital-splitting band is rather flat compared to the $E_{\mathrm{V}}$ top (in $k$ space) that exists in the band structure of $\mathrm{NiPS}_{3}$. Because linewidth broadening contributes more significantly than the energy shift with the change in the temperature in the $\mathrm{NiPS}_{3}$ lattice, the $\theta_{\text {iLO }}$ value is therefore greater than that of $\theta_{\mathrm{iB}}$ due to the larger averaged phonon temperature.

Transmittance and optical absorption of $\mathrm{NiPS}_{3}$ near band edge To further identify the band-edge nature, temperature-dependent transmittance $(T)$ measurement of a bulk NiPS 3 with a thickness of about $10 \mu \mathrm{m}$ was conducted. Figure $4 \mathrm{a}, \mathrm{b}$ show the transmittance absorption edge and $\mu$ TR spectra of the $A$ and $B$ excitons at 10 and $300 \mathrm{~K}$, respectively, together with a mathematical-derivative transmittance $(\Delta T)$ spectrum that is included for contrast. The transmittance spectrum of $\mathrm{NiPS}_{3}$ clearly reveals the presence of the $A_{1}, A_{2}$, and $E_{\infty}$ excitonic features at the absorption edge at $10 \mathrm{~K}$, similar to those detected in the $\mu \mathrm{TR}$ spectrum. In addition, the $\Delta T$ spectrum in Fig. 4a reveals the same energy position and line shape of the $A_{1}, A_{2}$, and $E_{\infty}$ features compared with those detected in the $\mu \mathrm{TR}$ measurement of $\mathrm{NiPS}_{3}$ at $10 \mathrm{~K}$. At $300 \mathrm{~K}$, the $A_{2}$ feature is ionized, and the energy value of $A_{1}$ from $\mu$ TR is about $1.366 \mathrm{eV}$. The transmittance edge is extremely close to the $A_{1}$ transition, and the $\Delta T$ spectral line shape is also similar to that of the $\mu T R$ feature. These results indicate that the energies of transmittance absorption edge (measured by $T$ ) and direct band edge (measured by $\mu \mathrm{TR}$ ) of bulk $\mathrm{NiPS}_{3}$ are very close. This result is dissimilar to other TMDCs such as $\mathrm{MoS}_{2}$, whose transmittance absorption edge is at $1.23-1.28 \mathrm{eV}$ (i.e., optical transition assisted by emission and absorption of phonons), while the direct gap is about $1.88 \mathrm{eV}$ in multilayer $\mathrm{MoS}_{2}{ }^{66}$. The energy difference between the indirect and direct bandgaps of $\mathrm{MoS}_{2}$ is about $0.6 \mathrm{eV}$. The weakened layer-by-layer decoupling of layered $\mathrm{NiPS}_{3}$ compared to $\mathrm{MoS}_{2}$ may be the main reason for the small energy difference between the indirect and direct bandgaps of the layered compound. Figure 4c, d show the temperature-dependent transmittance and absorption spectra of the $\mathrm{NiPS}_{3}$ sample from 10 to $300 \mathrm{~K}$. The absorption spectrum of $\mathrm{NiPS}_{3}$ at $10 \mathrm{~K}$ in Fig. $4 \mathrm{~d}$ is also similar to previous absorption curve that detected by antiferromagnet $\mathrm{NiPS}_{3}{ }^{58}$. The absorption edge in the temperaturedependent transmittance and absorption spectra demonstrates an energy blue-shift behavior with the decrease in the temperature from 300 to $10 \mathrm{~K}$, similar to the typical trend for semiconductors. The clear $A_{1}, A_{2}$, and $E_{\infty}$ transition features of the $A$ series excitons are still observed in the low-temperature absorption spectra $(T<180 \mathrm{~K})$, verifying the $\mu \mathrm{TR}$ results of fewlayer $\mathrm{NiPS}_{3}$ in Fig. 2a. At $300 \mathrm{~K}$, a shoulder peak of $E_{\infty} \approx 1.402 \mathrm{eV}$ is still observed for $\mathrm{NiPS}_{3}$ in Fig. $4 \mathrm{~d}$. This value is in good agreement with the direct band gap of the layered $\mathrm{NiPS}_{3}$ obtained using the Rydberg series as $E_{\infty} \approx A_{1}(1.366 \mathrm{eV})+E_{\mathrm{b}}{ }^{1}(36 \mathrm{meV})$ at room temperature.

Micro-photoluminescence result

To evaluate the origin of the band edge, thickness-dependent micro-photoluminescence $(\mu \mathrm{PL})$ measurements of layered $\mathrm{NiPS}_{3}$ were conducted at $300 \mathrm{~K}$. Figure $5 \mathrm{a}$ shows the $\mu \mathrm{PL}$ spectrum of a few-layer $\mathrm{NiPS}_{3}$ sample with a thickness of about $10 \mathrm{~nm}$. Two broadened peaks at about $E_{3 \mathrm{~d}} \approx 1.23 \mathrm{eV}$ and at $B \approx 1.825 \mathrm{eV}$ are observed in the $\mu \mathrm{PL}$ spectrum of few-layer $\mathrm{NiPS}_{3}$. The $\mathrm{E}_{3 \mathrm{~d}}$ peak is strong, but it gradually decreases with the increase of thickness in 

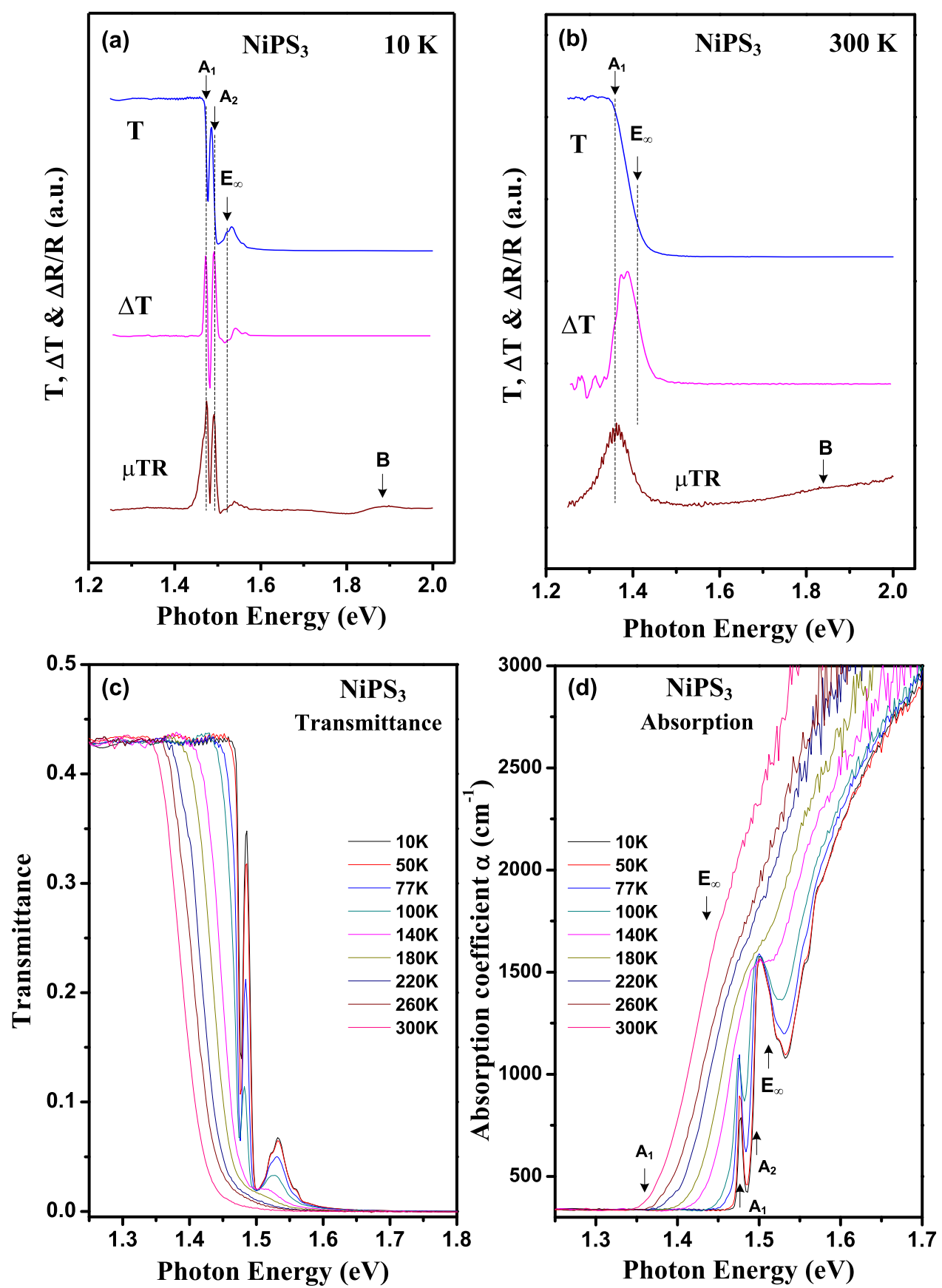

Fig. 4 The comparison of transmittance and $\boldsymbol{\mu T R}$ spectra in $\mathbf{N i P S}_{\mathbf{3}}$. Spectral comparison of the band-edge transitions in the transmittance $(T)$, first-derivative transmittance $(\Delta T)$, and $\mu \mathrm{TR}$ spectra at a $10 \mathrm{~K}$ and $\mathbf{b} 300 \mathrm{~K}$. The temperature-dependent transmittance and absorption spectra of the layered $\mathrm{NiPS}_{3}$ sample from 10 to $300 \mathrm{~K}$ are shown in $\mathbf{c}$ and d, respectively.

layered $\mathrm{NiPS}_{3}$ (Fig. 5b), whereas the $\mathrm{B}$ emission peak remains at a similar PL intensity with the change in the layer thickness from 10 to $200 \mathrm{~nm}$. A detailed analysis of the PL line-shape fitting of the PL spectrum in Fig. 5 a reveals that the $A_{1}$ peak (at $1.366 \mathrm{eV}$ ) is still involved in the broadened $E_{3 d}$ peak. The variation of PL intensity of the $E_{3 d}$ peak with changing the thickness of $\mathrm{NiPS}_{3}$ implies that multilayer $\mathrm{NiPS}_{3}$ is maybe an indirect semiconductor with an indirect-like emission of $\sim 1.23 \mathrm{eV}$, originating from the $\mathrm{Ni} 3 d \mathrm{e}_{\mathrm{g}}{ }^{*}$ band to the $E_{\mathrm{V}}$ transition assisted by phonons at $300 \mathrm{~K}$. The indirect-like emission of a 1.3-1.4 eV peak from indirect $E_{C}$ valley at $\Sigma$ point was usually observed in the multilayered $\mathrm{MoS}_{2}$ $(10-200 \mathrm{~nm})$ on $\mathrm{SiO}_{2} / \mathrm{Si}$ and on $\mathrm{Cu}$ substrate ${ }^{67}$. In the lightemission process, phonons are required to conserve the momentum, and photoexcited hot electrons are injection into the lower indirect valley of $E_{C}$ for resulting in indirect-like emission. As shown in Fig. 4b, the transmittance absorption edge of layered $\mathrm{NiPS}_{3}$ reveals that photons almost pass through at $1.23 \mathrm{eV}$, while this value starts to decrease until the photon energy becomes $1.366 \mathrm{eV}\left(A_{1}\right)$ at $300 \mathrm{~K}$. This result implies that the $\mathrm{Ni} 3 d e_{\mathrm{g}}{ }^{*}$ states might form an intermediate band located between the main $E_{C}$ $\left(\mathrm{P} 3 p^{*}+\mathrm{S} 3 p^{*}+\mathrm{Ni} 3 d^{*}\right)$ and the top of $E_{\mathrm{V}}$. The lower indirect-like emission bands of the $\mathrm{M} d$ to $d$ transitions of $\mathrm{M}_{2} \mathrm{P}_{2} \mathrm{~S}_{6}$ also can be observed in previous study of $\mathrm{Cd}_{2} \mathrm{P}_{2} \mathrm{~S}_{6}{ }^{68}$. The inset of Fig. $5 \mathrm{a}$ shows the probable band scheme of the band edge for PL band-edge emissions. The $E_{3 d}$ peak is an indirect-like emission caused by the intermediate band of $\mathrm{Ni} 3 d \mathrm{e}_{\mathrm{g}}{ }^{*}$ existed in $\mathrm{NiPS}_{3}$. The $A_{1}$ and $B$ 

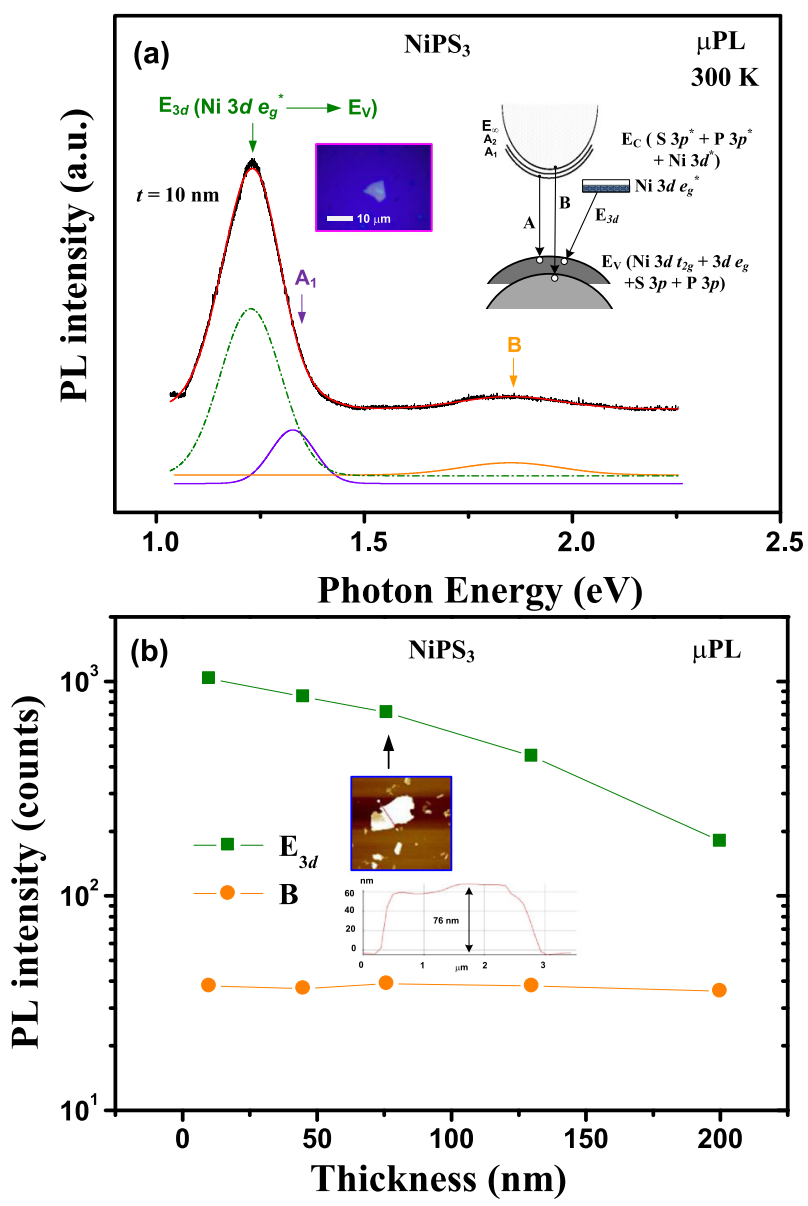

Fig. 5 Thickness-dependent $\mu \mathrm{PL}$ result of few-layer $\mathrm{NiPS}_{3}$ at $300 \mathrm{~K}$. a $\mu \mathrm{PL}$ spectrum of the band-edge emissions of a few-layer $\mathrm{NiPS}_{3}$ sample with a thickness of $\sim 10 \mathrm{~nm}$ at $300 \mathrm{~K}$. The fitting curves of the PL peaks are also included. The left inset shows a microscopic image of the few-layer sample, and the right inset shows the possible band-edge scheme for the observed PL emissions. b Thickness dependence of the PL intensities of the $E_{3 d}$ and $B$ peaks detected in a.

emissions are originated from the direct $E_{\mathrm{C}}$ bottom to the $E_{\mathrm{V}}$ top and to the spin-split-off band below $E_{\mathrm{V}}$, respectively.

\section{DISCUSSION}

In conclusion, the band-edge excitons of A series and B transition were clearly detected and observed in the high-quality few-layer $\mathrm{NiPS}_{3}$ grown by chemical vapor transport. The prominent excitonic sequence of $A_{1}, A_{2}$, and $E_{\infty}$ transitions and one bandto-band feature of $\mathrm{B}$ in $\mathrm{NiPS}_{3}$ were detected by micro-TR at $10 \mathrm{~K}$. The energy values and amplitudes of the excitonic sequence $A_{1}$, $A_{2}$, and $E_{\infty}$ followed a Rydberg series and continuum band. The excitonic binding energy of the $A_{1}$ level is about $36 \mathrm{meV}$ and its transition energy is $1.366 \mathrm{eV}$ at $300 \mathrm{~K}$. The temperature-dependent $\mu T R$ measurements of the $A$ series and $B$ transitions of the fewlayer $\mathrm{NiPS}_{3}$ were conducted to verify the thermal ionization energy of the excitons and the temperature-energy shift of the 2D material from 10 to $300 \mathrm{~K}$. The energy separation of the $A_{1}, A_{2}$, and $E_{\infty}$ transitions was in good agreement with the corresponding ionization temperatures to sustain the detected $\mathrm{A}$ series excitons in $\mathrm{NiPS}_{3}$. In addition, band-edge excitons of the $A_{1}, A_{2}$, and $E_{\infty}$ transitions were detected in the temperature-dependent transmittance and absorption spectra to identify the existence of the $A$ series excitons in the layered $\mathrm{NiPS}_{3}$. The direct optical gap of few-layer $\mathrm{NiPS}_{3}$ was determined to be $E_{\infty} \approx 1.402 \mathrm{eV}$ at $300 \mathrm{~K}$. The temperature-energy shift of the $A$ series excitons between 10 and $300 \mathrm{~K}$ was faster than those of $\mathrm{MoS}_{2}$ and $\mathrm{WS}_{2}$. From the $\mu \mathrm{PL}$ measurement of few-layer $\mathrm{NiPS}_{3}$, the indirect-like emission was observed at $1.23 \mathrm{eV}$, while a direct $\mathrm{B}$ band emission was observed at $1.825 \mathrm{eV}$. The indirect-like emission possibly originates from an intermediate band of $\mathrm{Ni} 3 d e_{\mathrm{g}}{ }^{*}$ to the $E_{\mathrm{V}}$ top recombination. The $A$ series excitons come from the top of $E_{\mathrm{V}}$ to the $E_{\mathrm{C}}$ bottom, while the $B$ feature is originated from the spin-split-off band to the $E_{\mathrm{C}}$ transition. Based on the experimental analysis, the origin of the band-edge excitons of the layered nickel phosphorus trisulfide was realized.

\section{METHODS}

\section{Sample growth}

Test samples were prepared by growing the layered single crystals of $\mathrm{NiPS}_{3}$ via CVT with $I_{2}$ as the transport agent. First, powdered elements of $\mathrm{Ni}$ (99.99\% purity), $P$ ( $99.999 \%$ purity), and S (99.999\% purity) were prepared. Second, the powder mixture of the starting materials and an appropriate amount of $I_{2}\left(10 \mathrm{mg} / \mathrm{cm}^{3}\right)$ were cooled with liquid nitrogen and sealed in a vacuum environment at $\sim 10^{-6}$ Torr inside a quartz ampoule. The growth temperature was set as $800^{\circ} \mathrm{C}$ (heating zone) $\rightarrow 700^{\circ} \mathrm{C}$ (growth zone) with a gradient of $-2.5^{\circ} \mathrm{C} / \mathrm{cm}$ for the simultaneous growth in two ampoules. The reaction occurred over $336 \mathrm{~h}$ to grow large single crystals. After this growth, several shiny sheet-like $\mathrm{NiPS}_{3}$ thick crystals with areas up to a scale of square centimeters were obtained. With the weakened van der Waals bonds between the layers, different layer thicknesses of $\mathrm{NiPS}_{3}$ can be mechanically exfoliated and transferred onto a $\mathrm{SiO}_{2} / \mathrm{Si}$ substrate using Scotch tapes with different stickiness properties.

\section{Microthermal-modulated reflectance}

For $\mu \mathrm{TR}$ measurements, a $150-\mathrm{W}$ tungsten halogen lamp served as the white light source. The white light source was dispersed by a $0.2-\mathrm{m}$ Photonics International (PTI) monochromator, which was equipped with a 1200 -grooves $/ \mathrm{mm}$ grating for providing monochromatic light. The $\mathrm{SiO}_{2} / \mathrm{Si}$ substrate decorated with few-layer $\mathrm{NiPS}_{3}$ (size of $0.8 \times 0.8 \times 0.01 \mathrm{~cm}^{3}$ ) was closely attached onto an Au-evaporated quartz plate. The monochromatic light source was coupled onto the few-layer or multilayer sample using a silica fiber, and it passed through a light-guiding microscope (LGM) equipped with an Olympus objective lens (50x, working distance $\sim 8 \mathrm{~mm}$ ). It served as the interconnection coupled medium between the few-layer sample and the incident and reflected lights ${ }^{69}$. The reflected light from the layered sample was collected by the LGM and then coupled to an EG\&G HUV200B Si detector using an additional silica fiber. Optical alignment was accomplished by the XYZ adjustment of the nano-flake sample using a CCD imaging camera in the LGM. A 4-Hz heating current $(\sim 0.5 \mathrm{~A})$ was periodically supplied to the Au heater for the thermal modulation of the lattice constant and band edge of the sample. Phase-sensitive detection was achieved by using an NF 5610B lock-in amplifier. A Janis open-circled liquid-helium cryostat equipped a Lakeshore 335 thermometer controller was used to facilitate temperature-dependent measurements from low temperatures to room temperature.

\section{Optical transmission measurement}

For the transmission measurement of bulk $\mathrm{NiPS}_{3}$ sample, the same monochromatic light source as that employed for $\mu \mathrm{TR}$ was used. The layer sample of $t \sim 10 \mu \mathrm{m}$ was closely mounted on a copper holder with a center hole of size approximately $500 \mu \mathrm{m}$ for light transmission. The incident light was chopped at $200 \mathrm{~Hz}$ with an optical chopper. The transmission light of the sample was passed to an EG\&G HUV200B Si photodetector. An NF $5610 B$ lock-in amplifier was used to implement the phase-sensitive photodetection. An RMC 22 close-cycled He compressor system that was equipped with a Lakeshore 335 thermometer controller facilitated the low temperature and temperature-dependent measurements.

\section{Micro-photoluminescence experiment}

The $\mu \mathrm{PL}$ measurements were conducted in an integrated RAMaker microscope spectrometer with a 532-nm solid-state diode-pump laser as the excitation source. The same LGM setup as that used for $\mu$ TR was utilized for the interconnection coupled medium between the few-layer 
$\mathrm{NiPS}_{3}$ sample, the incident and reflected (scattered) lights, and the chargecoupled-device (CCD) spectrometer. The laser power was $5 \mathrm{~mW}$, and the laser spot size was 3-5 $\mu \mathrm{m}$. The measurements were obtained from 1.1 to $2.25 \mathrm{eV}$.

\section{DATA AVAILABILITY}

The datasets generated during and/or analyzed during the current study are available from the corresponding author on reasonable request.

Received: 19 August 2020; Accepted: 1 December 2020; Published online: 05 January 2021

\section{REFERENCES}

1. Akinwande, D. et al. Graphene and two-dimensional materials for silicon technology. Nature 573, 507-518 (2019).

2. Lin, Z. et al. Solution-processable 2D semiconductors for high-performance largearea electronics. Nature 562, 254-258 (2018).

3. Wang, H., Zhang, X. \& Xie, Y. Recent progress in ultrathin two-dimensional semiconductors for photocatalysis. Mater. Sci. Eng. R. 130, 1-39 (2018).

4. Lin, Y. C. et al. Single-layer $\operatorname{ReS}_{2}$ : two-dimensional semiconductor with tunable inplane anisotropy. ACS Nano 9, 11249-11257 (2015).

5. Bae, S.-H. et al. Integration of bulk materials with two-dimensional materials for physical coupling and applications. Nat. Mater. 18, 550-560 (2019).

6. Radisavljevic, B., Radenovic, A., Brivio, J., Giacometti, V. \& Kis, A. Single-layer $\mathrm{MoS}_{2}$ transistors. Nat. Nanotechnol. 6, 147-150 (2011).

7. Ho, P. H. et al. High-mobility InSe transistors: the role of surface oxides. ACS Nano 11, 7362-7370 (2017).

8. Li, M. et al. High mobilities in layered InSe transistors with indium-encapsulationinduced surface charge doping. Adv. Mater. 30, 1803690 (2018).

9. Chuang, C. A., Lin, M. H., Yeh, B. X. \& Ho, C. H. Curvature-dependent flexible light emission from layered gallium selenide crystals. RSC Adv. 8, 2733 (2018).

10. Gao, L. Flexible device applications of 2D semiconductors. Small 13, 1603994 (2017).

11. Akinwande, D., Petrone, N. \& Hone, J. Two-dimensional flexible nanoelectronics. Nat. Commun. 5, 5678 (2014).

12. Mak, K. F., Lee, C., Hone, J., Shan, J. \& Heinz, T. F. Atomically thin $\mathrm{MoS}_{2}$ : a new direct-gap semiconductor. Phys. Rev. Lett. 105, 136805 (2010).

13. Mudd, G. W. et al. Tuning the bandgap of exfoliated InSe nanosheets by quantum confinement. Adv. Mater. 25, 5714-5718 (2013).

14. Sun, J. et al. Lateral $2 \mathrm{D} W \mathrm{WS}_{2} \mathrm{p}-\mathrm{n}$ homojunction formed by efficient chargecarrier-type modulation for high-performance optoelectronics. Adv. Mater. 32, 1906499 (2020).

15. Ho, C. H. Optical study of the structural change in $\mathrm{ReS}_{2}$ single crystals using polarized thermoreflectance spectroscopy. Opt. Express 13, 8-19 (2005).

16. Ovchinnikov, D. et al. Disorder engineering and conductivity dome in $\mathrm{ReS}_{2}$ with electrolyte gating. Nat. Commun. 7, 12391 (2016).

17. Zhou, X. et al. Rolling up $\mathrm{MoSe}_{2}$ nanomembranes as a sensitive tubular photodetector. Small 15, 1902528 (2019).

18. Ermolaev, G. A. et al. Broadband optical properties of monolayer and bulk $\mathrm{MoS}_{2}$ npj 2D Mater. Appl. 4, 21 (2020).

19. Ho, C. H., Wu, C. C. \& Cheng, Z. H. Crystal structure and electronic structure of $\mathrm{GaSe}_{1-x} \mathrm{~S}_{x}$ series layered solids. J. Cryst. Growth 279, 321-328 (2005).

20. $\mathrm{Wu}, \mathrm{C}$. C. et al. Optical properties of $\mathrm{GaSe}_{1-\mathrm{x}} \mathrm{S}_{\mathrm{x}}$ series layered semiconductors grown by vertical Bridgman method. Mater. Chem. Phys. 88, 313-317 (2004).

21. Ho, C. H. Thickness-dependent carrier transport and optically enhanced transconductance gain in III-VI multilayer InSe. 2D Mater. 3, 025019 (2016).

22. Cai, H. et al. Synthesis of highly anisotropic semiconducting GaTe nanomaterials and emerging properties enabled by epitaxy. Adv. Mater. 29, 1605551 (2017).

23. Ho, C. H., Chiou, M. C. \& Herninda, T. M. Nanowire grid polarization and polarized excitonic emission observed in multilayer GaTe. J. Phys. Chem. Lett. 11, 608-617 (2020)

24. Lee, K. C. et al. Analog circuit applications based on all-2D ambipolar ReSe, fieldeffect transistors. Adv. Funct. Mater. 29, 1809011 (2019).

25. $\mathrm{Ho}, \mathrm{C}$. $\mathrm{H}$. et al. Interplay between $\mathrm{Cr}$ dopants and vacancy clustering in the structural and optical properties of WSe $e_{2}$. ACS Nano 11, 11162-11168 (2017).

26. Chen, M. et al. Multibit data storage states formed in plasma-treated $\mathrm{MoS}_{2}$ transistors. ACS Nano 8, 4023-4032 (2014).

27. Yang, F. S. et al. Oxidation-boosted charge trapping in ultrasensitive van de Waals materials for artificial synaptic features. Nat. Commun. 11, 2972 (2020).

28. Ho, C. H., Hsieh, M. H. \& Wu, C. C. Photoconductance and photoresponse of layer compound photodetectors in the UV-visible region. Rev. Sci. Instrum. 77, 113102 (2006)
29. Jung, C. S. et al. Photoluminescence and photocurrents of $\mathrm{GaS}_{1-\mathrm{x}} \mathrm{Se}_{\mathrm{x}}$ nanobelts. Chem. Mater. 28, 5811-5820 (2016).

30. Ho, C. H. \& Huang, K. W. Visible luminescence and structural property of $\mathrm{GaSe}_{1-x} \mathrm{~S}_{x}$ $(0 \leq x \leq 1)$ series layered crystals. Solid Stat. Commun. 136, 591-594 (2005).

31. Wang, F. et al. New frontiers on van der Waals layered metal phosphorous trichalcogenides. Adv. Funct. Mater. 28, 1802151 (2018).

32. Susner, M. A., Chyasnavichyus, M., McGuire, M. A., Ganesh, P. \& Maksymovych, P. Metal thio- and selenophosphates as multifunctional van der Waals layered materials. Adv. Mater. 29, 1602852 (2017).

33. Liang, Q. et al. General and scalable solid-state synthesis of $2 \mathrm{D} M \mathrm{MPS}_{3}(\mathrm{M}=\mathrm{Fe}, \mathrm{Co}$, $\mathrm{Ni}$ ) nanosheets and tuning their $\mathrm{Li} / \mathrm{Na}$ storage properties. Small Methods $\mathbf{1}$, 1700304 (2017).

34. Sivadas, N., Okamoto, S. \& Xiao, D. Gate-controllable magneto-optic Kerr effect in layered collinear antiferromagnets. Phys. Rev. Lett. 117, 267203 (2016)

35. Joy, P. A. \& Vasudevan, S. Magnetism in the layered transition-metal thiophosphates $\mathrm{MPS}_{3}$ (M=Mn, Fe, and Ni). Phys. Rev. B 46, 5425-5433 (1992).

36. $\mathrm{Chu}$, J. et al. High-performance ultraviolet photodetector based on a few-layered 2D NiPS 3 nanosheet. Adv. Funct. Mater. 27, 1701342 (2017).

37. Wang, J. et al. Activating basal planes of $\mathrm{NiPS}_{3}$ for hydrogen evolution by nonmetal heteroatom doping. Adv. Funct. Mater. 30, 1908708 (2020)

38. Zhang, X., Zhang, S., Li, J. \& Wang, E. One-step synthesis of well-structured $\mathrm{NiS}-\mathrm{Ni}_{2} \mathrm{P}_{2} \mathrm{~S}_{6}$ nanosheets on nickel foam for efficient overall water splitting. $J$. Mater. Chem. A 5, 22131-22136 (2017).

39. Jenjeti, R. N., Kumar, R. \& Sampath, S. Two-dimensional, few-layer $\mathrm{NiPS}_{3}$ for flexible humidity sensor with high selectivity. J. Mater. Chem. A 7, 14545-14551 (2019).

40. Jenjeti, R. N., Kumar, R., Austeria, M. P. \& Sampath, S. Field-effect transistor based on layered NiPS . Sci. Rep. 8, 8586 (2018).

41. Brec, R., Schleich, D. M., Ouvrard, G., Louisy, A. \& Rouxel, J. Physical properties of lithium intercalation compounds of the layered transition chalcogenophosphates. Inorg. Chem. 18, 1814-1818 (1979).

42. Mueller, T. \& Malic, E. Exciton physics and device application of two-dimensional transition metal dichalcogenide semiconductors. npj 2D Mater. Appl. 2, 29 (2018).

43. Ho, C. H., Wu, C. S., Huang, Y. S., Liao, P. C. \& Tiong, K. K. Temperature dependence of energies and broadening parameters of the band-edge excitons of $\mathrm{Mo}_{1-\mathrm{x}} \mathrm{W}_{\mathrm{x}} \mathrm{S}_{2}$ single crystals. J. Phys. 10, 9317-9328 (1998)

44. Ho, C. H. \& Liu, Z. Z. Complete-series excitonic dipole emissions in few layer $\mathrm{ReS}_{2}$ and $\mathrm{ReSe}_{2}$ observed by polarized photoluminescence spectroscopy. Nano Energy 56, 641-650 (2019)

45. Ho, C. H., Yen, P. C., Huang, Y. S. \& Tiong, K. K. Photoreflectance study of the excitonic transitions of rhenium disulphide layer compounds. Phys. Rev. B 66 245207 (2002).

46. Ho, C. H. \& Huang, C. E. Optical property of the near band-edge transitions in rhenium disulfide and diselenide. J. Alloy Compd 383, 74-79 (2004).

47. Taylor, B., Steger, J. \& Wold, A. Preparation and properties of some transition metal phosphorus trisulfide compounds. J. Solid Stat. Chem. 7, 461-467 (1973).

48. Foot, P. J. S. \& Shaker, N. G. Amine intercalates of lamellar compounds NiPS3 and CdPS3 Mater. Res. Bull. 18, 173-180 (1983).

49. Pollak, F. H. \& Shen, H. Modulation spectroscopy of semiconductors: bulk/thin film, microstructures, surfaces/interfaces and devices. Mater. Sci. Eng. $R$ 10, 275-374 (1993)

50. Ho, C. H., Tseng, C. Y. \& Tien, L. C. Thermoreflectance characterization of $\beta-\mathrm{Ga}_{2} \mathrm{O}_{3}$ thin-film nanostrips. Opt. Express 18, 16360-16369 (2010).

51. Ho, C. H., Tsai, M. C. \& Wong, M. S. Characterization of indirect and direct inter band transitions of anatase $\mathrm{TiO}_{2}$ by thermoreflectance spectroscopy. Appl. Phys. Lett. 93, 081904 (2008).

52. Aspnes, D. E. in Handbook on Semiconductors vol. 2 (ed. Balkanski, M.) Chap. 4A, 109-154 (North Holland, Amsterdam, 1980).

53. Chernikov, A. et al. Exciton binding energy and nonhydrogenic Rydberg series in monolayer WS 2 . Phys. Rev. Lett. 113, 076802 (2014).

54. Aivazian, G. et al. Many-body effect in nonlinear optical responses of 2D layered semiconductors. 2D Mater. 4, 025024 (2017).

55. Yazyev, O. V. \& Kis, A. MoS 2 and semiconductors in the flatband. Mater. Today 18, 20-30 (2015).

56. Yu, P. Y. \& Cardona, M. in Fundamentals of Semiconductors, 3rd edn, 288 (Springer, New York, 2001).

57. Khumalo, F. S. \& Hughes, H. P. Reflectance spectra of some FePS 3 -type layer compounds in the vacuum ultraviolet. Phys. Rev. B 23, 5375-5383 (1981).

58. Kang, S. et al. Coherent many-body exciton in van der Waals antiferromagnets $\mathrm{NiPS}_{3}$. Nature 583, 785-789 (2020).

59. Grasso, V., Neri, F., Santangelo, S., Silipigni, L. \& Piacentini, M. Electronic transport properties of $\mathrm{NiPS}_{3}$. Phys. Rev. B 37, 4419-4424 (1988).

60. Piacentini, M., Khumalo, F. S., Olson, C. G., Anderegg, J. W. \& Lynch, D. W. Optical transitions, XPS, and electronic states in NiPS 3 . Chem. Phys. 65, 289-304 (1982) 
61. Kim, S. Y. et al. Charge-spin correlation in van der Waals antiferromagnet $\mathrm{NiPS}_{3}$. Phys. Rev. Lett. 120, 136402 (2018).

62. Kadantsev, E. S. \& Hawrylakb, P. Electronic structure of a single $\mathrm{MoS}_{2}$ monolayer. Solid State Commun. 152, 909-913 (2012).

63. Steinhoff, A. et al. Efficient excitonic photoluminescence in direct and indirect band gap monolayer MoS 2 . Nano Lett. 15, 6841-6847 (2015).

64. Lantenschlager, P., Garriga, M., Logothetidis, S. \& Cardona, M. Interband critical points of GaAs and their temperature dependence. Phy. Rev. B 35, 9174-9189 (1987).

65. Malikova, L. et al. Temperature dependence of the direct gaps of $\mathrm{ZnSe}$ and $\mathrm{Zn}_{0.56} \mathrm{Cd}_{0.44}$ Se. Phys. Rev. B 54, 1819-1824 (1996).

66. Kopaczek, J. et al. Direct and indirect optical transitions in bulk and atomically thin $\mathrm{MoS}_{2}$ studied by photoreflectance and photoacoustic spectroscopy. J. Appl. Phys. 125, 135701 (2019).

67. Li, Z. et al. Indirect band gap emission by hot electron injection in metal/MoS and metal/WSe $\mathrm{W}_{2}$ heterojunctions. Nano Lett. 15, 3977-3982 (2015).

68. Cleary, D. A. \& Francis, A. H. Photoluminescence study of layered transition metal phosphourus chalcogenides and their pyridine intercalation compounds. J. Limin. 35, 163-170 (1986).

69. Ho, C. H. \& Li, J. X. Polarized band-edge emission and dichroic optical behavior in thin multilayer GeS. Adv. Opt. Mater. 5, 1600814 (2017).

\section{ACKNOWLEDGEMENTS}

The authors would like to acknowledge the funding support from the Ministry of Science and Technology, Taiwan under the grant numbers MOST 107-2112-M-011001-MY3 and MOST 108-2221-E-011-138-MY2.

\section{AUTHOR CONTRIBUTIONS}

C.H.H. conceived the idea and conducted the experiments. T.Y.H. grew the samples and performed the optical measurements. L.C.M. did the micro-PL experiment. C.H.H. also analyzed (calculated) the measurement data, and wrote the manuscript.

\section{COMPETING INTERESTS}

The authors declare no competing interests.

\section{ADDITIONAL INFORMATION}

Supplementary information is available for this paper at https://doi.org/10.1038/ s41699-020-00188-8.

Correspondence and requests for materials should be addressed to C.-H.H.

Reprints and permission information is available at http://www.nature.com/ reprints

Publisher's note Springer Nature remains neutral with regard to jurisdictional claims in published maps and institutional affiliations.

(i) Open Access This article is licensed under a Creative Commons adaptation, distribution and reproduction in any medium or format, as long as you give appropriate credit to the original author(s) and the source, provide a link to the Creative Commons license, and indicate if changes were made. The images or other third party material in this article are included in the article's Creative Commons license, unless indicated otherwise in a credit line to the material. If material is not included in the article's Creative Commons license and your intended use is not permitted by statutory regulation or exceeds the permitted use, you will need to obtain permission directly from the copyright holder. To view a copy of this license, visit http://creativecommons. org/licenses/by/4.0/.

(c) The Author(s) 2021 